The Astrophysical Journal, Submitted 2002 May

\title{
SCREENING CORRECTIONS TO THE ELECTRON CAPTURE RATES IN DENSE STARS BY THE RELATIVISTICALLY DEGENERATE ELECTRON LIQUID
}

\author{
Naoki Itoh, Nami Tomizawa, Masaya Tamamura, and Shinya Wanajo \\ Department of Physics, Sophia University, 7-1 Kioi-cho, Chiyoda-ku, Tokyo, 102-8554, \\ Japan; \\ n_itoh@sophia.ac.jp,tomiza-n@sophia.ac.jp,m-tamamu@sophia.ac.jp,wanajo@sophia.ac.jp \\ and \\ SATOSHI NozAWA \\ Josai Junior College for Women, 1-1 Keyakidai, Sakado-shi, Saitama, 350-0295, Japan; \\ snozawa@josai.ac.jp \\ The Astrophysical Journal, Submitted 2002 May
}

\begin{abstract}
We calculate the screening corrections to the electron capture rates in dense stars by the relativistically degenerate electron liquid. In order to calculate the screening corrections we adopt the linear response theory which is widely used in the field of solid state physics and liquid metal physics. In particular, we use the longitudinal dielectric function for the relativistically degenerate electron liquid derived by Jancovici. We calculate the screening potential at the position of the nucleus. By using this screening potential one can calculate the screening corrections to the electron capture rates. We will present accurate analytic fitting formulae which summarize our numerical results. These fitting formulae will facilitate the application of the present results. The screening corrections to the electron capture rates are typically a few percent.
\end{abstract}

Subject headings: nuclear reactions: electron captures — stars: interiors 


\section{INTRODUCTION}

Since the pioneering works of Fuller, Fowler, \& Newman (1980, 1982a, 1982b, 1985), calculations of stellar weak-interaction rates have entered an era of precision science. More recently an authoritative work of Langanke \& Martinez-Pinedo (2000) on this subject appeared.

Since the presupernova stellar evolution and stellar nucleosynthesis critically depend on the details of the stellar weak-interaction rates (e.g., Wanajo et al. 2002), it is extremely important to calculate accurately the screening corrections to the electron capture rates in dense stars by the relativistically degenerate electron liquid.

This problem has been already addressed by many authors (Couch \& Loumos 1974; Takahashi, El Eid, \& Hillebrandt 1978; Gutierrez et al. 1996; Luo \& Peng 1996; Bravo \& Garcia-Senz 1999). The plasma effects on the chemical potential of the nucleus and hence on the threshold energy for the electron capture, in particular, have been discussed by Couch \& Loumos (1974), Gutierrez et al. (1996), as well as by Bravo \& Garcia-Senz (1999).

In this paper we will address ourselves to the calculation of the effective potential energy felt by the relativistically degenerate electron. We will use the linear response theory in order to calculate the screening potential caused by the relativistically degenerate electron liquid.

The present paper is organized as follows. In $\S 2$ we will calculate the effective potential energy felt by the electron using the longitudinal dielectric function of the relativistically degenerate electron liquid derived by Jancovici (1962). We will thereby calculate the screening potential which will be used for the calculation of the screening corrections to the electron capture rates. In $\S 3$ we will summarize the numerical results in the form of analytic fitting formulae which will facilitate the application of the present results. We will give concluding remarks in $\S 4$.

\section{SCREENING OF THE COULOMB POTENTIAL BY THE RELATIVISTICALLY DEGENERATE ELECTRON LIQUID}

The Coulomb corrections to the beta decay rates have been discussed in many standard textbooks on beta decay (e.g., Morita 1973). Some authors (Takahashi, El Eid, \& Hillebrandt 1978; Fuller, Fowler, \& Newman 1980) have discussed possible importance of the screening effects on the electron capture rates at extremely high densities. Therefore, it is extremely important to calculate accurately the screening corrections to the electron capture rates by the relativistically degenerate electron liquid. 
We consider the density-temperature regime in which the electrons are strongly degenerate. This condition is expressed as (Itoh et al. 1983)

$$
T \ll T_{F}=5.930 \times 10^{9}\left[\left\{1+1.018(Z / A)^{2 / 3} \rho_{6}^{2 / 3}\right\}^{1 / 2}-1\right][\mathrm{K}]
$$

where $T_{F}$ is the electron Fermi temperature, $\rho_{6}$ is the mass density in units of $10^{6} \mathrm{~g} \mathrm{~cm}^{-3}$, and $Z$ and $A$ are the atomic number and mass number of the nucleus considered, respectively. We assume complete pressure ionization. Therefore, no bound electrons will exist around the nucleus. This assumption is valid when the following condition is satisfied (Itoh et al. 1996):

$$
\frac{Z}{A} \rho \geq 0.378 Z^{3}\left[\mathrm{~g} \mathrm{~cm}^{-3}\right]
$$

where $\rho$ is the mass density.

The potential energy of the electron due to the pure Coulomb potential $-Z e^{2} / r$ is changed to $V(r)$ because of the screening by the relativistically degenerate electron liquid. In this paper we use the static longitudinal dielectric function due to the relativistically degenerate electron liquid calculated by Jancovici (1962) based on the relativistic randomphase approximation. Jancovici's static longitudinal dielectric function is written in the form (Itoh et al. 1984)

$$
\begin{aligned}
\epsilon(q, 0)= & 1+\left(\frac{2}{3 \pi^{2}}\right)^{2 / 3} \frac{r_{s}}{q^{2}}\left[\frac{2}{3}\left(1+x^{2}\right)^{1 / 2}-\frac{2 q^{2} x}{3} \sinh ^{-1} x\right. \\
& +\left(1+x^{2}\right)^{1 / 2} \frac{x^{2}+1-3 q^{2} x^{2}}{6 q x^{2}} \ln \left|\frac{1+q}{1-q}\right| \\
& \left.+\frac{2 q^{2} x^{2}-1}{6 q x^{2}}\left(1+q^{2} x^{2}\right)^{1 / 2} \ln \left|\frac{q\left(1+x^{2}\right)^{1 / 2}+\left(1+q^{2} x^{2}\right)^{1 / 2}}{q\left(1+x^{2}\right)^{1 / 2}-\left(1+q^{2} x^{2}\right)^{1 / 2}}\right|\right], \\
q= & \frac{k}{2 k_{F}}, \\
x= & \frac{\hbar k_{F}}{m_{e} c}=\frac{1}{137.036}\left(\frac{9 \pi}{4}\right)^{1 / 3} r_{s}^{-1}, \\
r_{s}= & \frac{a_{e}}{\hbar^{2}}=1.388 \times 10^{-2}\left(\frac{A}{Z \rho_{6}}\right)^{1 / 3}, \\
& \frac{4}{m_{e} e^{2}} \\
& \pi a_{e}^{3} n_{e}=1,
\end{aligned}
$$

where $n_{e}$ is the electron number density. The electron Fermi wavenumber is expressed as

$$
k_{F}=2.613 \times 10^{10}\left(\frac{Z}{A} \rho_{6}\right)^{1 / 3}\left[\mathrm{~cm}^{-1}\right] \text {. }
$$


The ion-sphere radius $a_{i}$ is written as (Itoh \& Kohyama 1993)

$$
\begin{aligned}
\frac{4}{3} \pi a_{i}^{3} n_{i} & =1 \\
a_{i} & =0.7346 \times 10^{-10}\left(\frac{\rho_{6}}{A}\right)^{-1 / 3}[\mathrm{~cm}],
\end{aligned}
$$

where $n_{i}$ is the ion number density. We also have the relationship

$$
k_{F} a_{i}=\left(\frac{9 \pi}{4}\right)^{1 / 3} Z^{1 / 3} .
$$

The potential energy of the electron $V(r)$ which takes into account the screening by the relativistially degenerate electron liquid is written as

$$
\begin{aligned}
V(r) & =-\frac{Z e^{2}}{2 \pi^{2}} \int \frac{e^{i \vec{k} \cdot \vec{r}}}{k^{2} \epsilon(k, 0)} d^{3} k \\
& =-\frac{Z e^{2}\left(2 k_{F}\right)}{2 k_{F} r} \frac{2}{\pi} \int_{0}^{\infty} \frac{\sin \left[\left(2 k_{F} r\right) q\right]}{q \epsilon(q, 0)} d q
\end{aligned}
$$

Therefore the increment of the potential energy due to the screening by the relativistically degenerate electron liquid is

$$
\begin{aligned}
V_{s}(r) & \equiv V(r)-\left(-\frac{Z e^{2}}{r}\right) \\
& =Z e^{2}\left(2 k_{F}\right) \frac{1}{2 k_{F} r}\left\{1-\frac{2}{\pi} \int_{0}^{\infty} \frac{\sin \left[\left(2 k_{F} r\right) q\right]}{q \epsilon(q, 0)} d q\right\} \\
& =7.525 \times 10^{-3} Z\left(\frac{Z}{A} \rho_{6}\right)^{1 / 3}[\mathrm{MeV}] \frac{1}{2 k_{F} r}\left\{1-\frac{2}{\pi} \int_{0}^{\infty} \frac{\sin \left[\left(2 k_{F} r\right) q\right]}{q \epsilon(q, 0)} d q\right\}
\end{aligned}
$$

Equation (2.12) is based on the linear response theory (e.g., Pines \& Nozières 1966). Dharma-wardama \& Perrot $(1982,1990)$ have carried out the density-functional study of hydrogen plasmas as well as the density-functional study of C, Si, and Ge metallic liquids and have found that the detailed results of the density-functional calculations of these systems are close to the results obtained by the linear response theory. They have also confirmed that the density-functional theory as well as the linear response theory reproduce satisfactorily the experimental results on the Ge metallic liquid, thereby proving the excellence of these theories for this system. This fact gives great support to our use of the linear response theory in the present problem.

Compared with the case of the pure Coulomb potential, the effective electron energy at the position of the nucleus is increased by $V_{s}(0)$. Hence the usual factor in the 
electron-capture rates $p E F(Z, E)$ (Morita 1973; Fuller, Fowler, \& Newman 1980; Langanke $\&$ Martinez-Pinedo 2000) should be replaced by

$$
\left\{\left[E-V_{s}(0)\right]^{2}-m_{e}^{2}\right\}^{1 / 2}\left[E-V_{s}(0)\right] F\left[Z, E-V_{s}(0)\right]
$$

At the same time the shift in the threshold energy for the electron capture should be taken into account (Couch \& Loumos 1974; Gutierrez et al. 1996; Bravo \& Garcia-Senz 1999).

In Figure 1 we show the function

$$
I \equiv \frac{2}{\pi} \int_{0}^{\infty} \frac{\sin \left[\left(2 k_{F} r\right) q\right]}{q \epsilon(q, 0)} d q
$$

as a function of $R=2 k_{F} r$ for various values of $r_{s}$. In Figure 2 we show the function

$$
J \equiv \frac{1}{2 k_{F} r}\left\{1-\frac{2}{\pi} \int_{0}^{\infty} \frac{\sin \left[\left(2 k_{F} r\right) q\right]}{q \epsilon(q, 0)} d q\right\}
$$

as a function of $R=2 k_{F} r$ for various values of $r_{s}$. In Figure 3 we enlarge Figure 2 for small values of $R=2 k_{F} r$. In Figure 4 we show $J(R=0)$ as a function of $r_{s}$. Equation (2.13) can be rewritten as

$$
V_{s}(r)=7.525 \times 10^{-3} Z\left(\frac{Z}{A} \rho_{6}\right)^{1 / 3} J[\mathrm{MeV}]
$$

The nuclear radius $r_{n u c}$ can be expressed as (Morita 1973)

$$
r_{n u c}=1.2 A^{1 / 3}[\mathrm{fm}] .
$$

From equations (2.8) and (2.18) we have

$$
2 k_{F} r_{n u c}=6.3 \times 10^{-3} Z^{1 / 3} \rho_{6}^{1 / 3} .
$$

From Figure 3 we see that the function $J(R)$ is almost constant for small values of $R$ which corresponds to $2 k_{F} r_{n u c}$ given by equation (2.19), when the mass density is not extremely high. This fact justifies our use of $V_{s}(0)$ for the screening potential at the nuclear radius. When the mass density becomes extremely high, then one should use the value of the screening potential $V_{s}(r)$ by adopting the value of the function $J\left(R=2 k_{F} r_{n u c}\right)$.

The electron Fermi energy is given by

$$
E_{F}=0.5110\left[\left\{1+1.018(Z / A)^{2 / 3} \rho_{6}^{2 / 3}\right\}^{1 / 2}-1\right][\mathrm{MeV}]
$$


When the electrons are extremely relativistic, we have

$$
\frac{V_{s}(r)}{E_{F}} \simeq 1.460 \times 10^{-2} Z J
$$

Therefore, we find that the screening potential at the origin $V_{s}(0)$ is typically a few percent of the electron Fermi energy. Thus we conclude that the screening corrections to the electron capture rates by the relativistically degenerate electron liquid are not as great as anticipated by Takahashi, El Eid, \& Hillebrandt (1978) and also by Fuller, Fowler, \& Newman (1980).

In passing, it is interesting to compare our present detailed calculation with the FermiThomas model which is generally more crude than the random-phase approximation that has been adopted in the present paper (Pines and Nozières 1966). In the Fermi-Thomas model the screening potential at the origin is expressed as (Pines and Nozières 1966)

$$
\left[V_{s}(0)\right]_{F T}=Z e^{2} k_{F T}
$$

where $k_{F T}$ is the Fermi-Thomas screening wave number. When the electrons are extremely relativistic, the Fermi-Thomas screening wave number is expressed as (Flowers \& Itoh 1976)

$$
\frac{k_{F T}}{2 k_{F}} \simeq \frac{1}{2}\left(\frac{4}{\pi} \frac{1}{137.036}\right)^{1 / 2}=0.0482
$$

By Comparing with Figures 2, 3, 4, we find that the prediction of the Fermi-Thomas model is quite good (the accuracy is about 7\%) for the present problem when the electrons are extremely relativistic.

\section{ANALYTIC FITTING FORMULAE}

In this section we will present accurate analytic fitting formulae for $J(R)$ in order to facilitate the application of the numerical results obtained in the present paper. We have carried out the numerical calculations of $J(R)$ for $0.00001 \leq r_{s} \leq 0.1,0 \leq R \leq 50.0$. We express the analytic fitting formula by

$$
\begin{aligned}
J\left(r_{s}, R\right) & =\sum_{i, j=0}^{10} a_{i j} s^{i} u^{j}, \\
s & \equiv \frac{1}{2}\left(\log _{10} r_{s}+3\right), \\
u & \equiv \frac{1}{25.0}(R-25.0) .
\end{aligned}
$$


The coefficients $a_{i j}$ are presented in Table 1. The accuracy of the fitting is generally better than $0.1 \%$.

The value of $J\left(r_{s}, R\right)$ for $R=0$ is of course obtained by the fitting formulae (3.1), (3.2), (3.3). For the sake of simplicity we will give a separate fitting formula

$$
\begin{aligned}
J\left(r_{s}, R=0\right) & =\sum_{i=0}^{10} b_{i} s^{i}, \\
s & \equiv \frac{1}{2}\left(\log _{10} r_{s}+3\right) .
\end{aligned}
$$

The coefficients $b_{i}$ are presented in Table 2 . The accuracy of the fitting is generally better than $0.1 \%$.

\section{CONCLUDING REMARKS}

We have studied the screening corrections to the electron capture rates by the relativistically degenerate electron liquid. In particular, we have calculated the screening potential caused by the relativistically degenerate electron by using Jancovici's (1962) static longitudinal dielectric function. We have found that the screening potential is typically a few percent of the electron Fermi energy. Hence we conclude that the screening corrections to the electron capture rates at high densities are not as great as anticipated by Takahashi, El Eid, \& Hillebrandt (1978) and also by Fuller, Fowler, \& Newman (1980).

We have presented accurate analytic fitting formulae which will be useful when one wishes to apply the present results to the calculations of the screening corrections to the electron capture rates at high densities.

We wish to thank K. Nomoto for drawing our attention to the present important problem. We are grateful to Y. Takada for clarifying the validity of the linear response theory in the present problem. We also thank Y. Oyanagi for allowing us to use the least-squares fitting program SALS. This work is financially supported in part by Grants-in-Aid of the Japanese Ministry of Education, Culture, Sports, Science, and Technology under contracts $13640245,13740129$. 


\section{REFERENCES}

Bravo, E., \& Garcia-Senz, D. 1999, MNRAS, 307, 984

Couch, R. G., \& Loumos, G. L. 1974, ApJ, 194, 385

Dharma-wardana, M. W. C., \& Perrot, F. 1982, Phys. Rev., A26, 2096

Dharma-wardana, M. W. C., \& Perrot, F. 1990, Phys. Rev. Letters, 65, 76

Flowers, E., \& Itoh, N. 1976, ApJ, 206, 218

Fuller, G. M., Fowler, W. A., \& Newman, M. J. 1980, ApJS, 42, 447

Fuller, G. M., Fowler, W. A., \& Newman, M. J. 1982a, ApJ, 252, 715

Fuller, G. M., Fowler, W. A., \& Newman, M. J. 1982b, ApJS, 48, 279

Fuller, G. M., Fowler, W. A., \& Newman, M. J. 1985, ApJ, 293, 1

Gutierrez, J., Garcia-Berro, E., Iben, I., Isern, J., Labay, J., \& Canal, R. 1996, ApJ, 459, 701

Itoh, N., Hayashi, H., Nishikawa, A., \& Kohyama, Y. 1996, ApJS, 102, 411

Itoh, N., \& Kohyama, Y. 1993, ApJ, 404, 268

Itoh, N., Kohyama, Y., Matsumoto, M., \& Seki, M. 1984, ApJ, 285, 758 (erratum 404, 418 [1993])

Itoh, N., Mitake, S., Iyetomi, H., \& Ichimaru, S. 1983, ApJ, 273, 774

Jancovici, B. 1962, Nuovo Cimento, 25, 428

Langanke, K., \& Martinez-Pinedo 2000, Nucl. Phys., A673, 481

Luo, Z. Q., \& Peng, Q. H. 1996, Science in China (Series A), 39, 776

Morita, M. 1973, Beta Decay and Muon Capture (Reading, Massachusetts: W. A. Benjamin)

Pines, D., \& Nozières, P. 1966, The Theory of Quantum Liquids (New York: W. A. Benjamin)

Takahashi, K., El Eid, M. F., \& Hillebrandt, W. 1978, A \& A, 67, 185

Wanajo, S., Itoh, N., Ishimaru, Y., Nozawa, S., \& Beers, T. C. 2002, submitted to ApJ 
Table 1: Coefficients $a_{i j}$

\begin{tabular}{rrrrrr}
\hline & $j=0$ & \multicolumn{1}{c}{$j=1$} & $j=2$ & $j=3$ & $j=4$ \\
\hline$i=0$ & $2.80066 \mathrm{E}-2$ & $-1.34650 \mathrm{E}-2$ & $4.70157 \mathrm{E}-3$ & $-1.62773 \mathrm{E}-3$ & $3.57498 \mathrm{E}-4$ \\
$i=1$ & $2.91425 \mathrm{E}-4$ & $-4.77037 \mathrm{E}-4$ & $3.24480 \mathrm{E}-4$ & $-1.84976 \mathrm{E}-4$ & $1.26065 \mathrm{E}-4$ \\
$i=2$ & $3.71730 \mathrm{E}-4$ & $-1.11205 \mathrm{E}-3$ & $1.11414 \mathrm{E}-3$ & $-7.49702 \mathrm{E}-4$ & $4.08871 \mathrm{E}-4$ \\
$i=3$ & $-3.40043 \mathrm{E}-4$ & $1.76471 \mathrm{E}-3$ & $-7.27685 \mathrm{E}-4$ & $-2.02979 \mathrm{E}-3$ & $3.21763 \mathrm{E}-3$ \\
$i=4$ & $8.38363 \mathrm{E}-3$ & $-3.40534 \mathrm{E}-3$ & $-1.66683 \mathrm{E}-3$ & $-4.20858 \mathrm{E}-3$ & $1.00310 \mathrm{E}-2$ \\
$i=5$ & $2.98675 \mathrm{E}-2$ & $-3.93466 \mathrm{E}-2$ & $1.81463 \mathrm{E}-2$ & $-6.64180 \mathrm{E}-3$ & $9.00760 \mathrm{E}-3$ \\
$i=6$ & $1.44775 \mathrm{E}-2$ & $-4.06027 \mathrm{E}-2$ & $3.23311 \mathrm{E}-2$ & $-8.65196 \mathrm{E}-3$ & $-2.12560 \mathrm{E}-3$ \\
$i=7$ & $-3.96957 \mathrm{E}-2$ & $4.02288 \mathrm{E}-2$ & $-5.91508 \mathrm{E}-3$ & $-8.13296 \mathrm{E}-3$ & $-1.31089 \mathrm{E}-4$ \\
$i=8$ & $-3.32815 \mathrm{E}-2$ & $5.69571 \mathrm{E}-2$ & $-3.05845 \mathrm{E}-2$ & $-1.66852 \mathrm{E}-3$ & $7.99359 \mathrm{E}-3$ \\
$i=9$ & $1.51205 \mathrm{E}-2$ & $-1.18443 \mathrm{E}-2$ & $-2.51457 \mathrm{E}-3$ & $4.25544 \mathrm{E}-3$ & $2.57406 \mathrm{E}-3$ \\
$i=10$ & $1.52622 \mathrm{E}-2$ & $-2.14839 \mathrm{E}-2$ & $8.10578 \mathrm{E}-3$ & $2.56649 \mathrm{E}-3$ & $-1.54054 \mathrm{E}-3$ \\
\hline \hline & $j=6$ & $j=7$ & $j=8$ & $j=9$ & $j=10$ \\
\hline$i=0$ & $-1.84849 \mathrm{E}-3$ & $-5.69873 \mathrm{E}-3$ & $4.05787 \mathrm{E}-3$ & $4.57584 \mathrm{E}-3$ & $-3.62782 \mathrm{E}-3$ \\
$i=1$ & $-4.25228 \mathrm{E}-4$ & $-5.70927 \mathrm{E}-4$ & $6.77612 \mathrm{E}-4$ & $3.74132 \mathrm{E}-4$ & $-4.07058 \mathrm{E}-4$ \\
$i=2$ & $-7.64487 \mathrm{E}-4$ & $-1.83728 \mathrm{E}-3$ & $1.70005 \mathrm{E}-3$ & $1.40476 \mathrm{E}-3$ & $-1.30179 \mathrm{E}-3$ \\
$i=3$ & $-7.73692 \mathrm{E}-3$ & $-1.08840 \mathrm{E}-2$ & $1.41242 \mathrm{E}-2$ & $6.32205 \mathrm{E}-3$ & $-7.84898 \mathrm{E}-3$ \\
$i=4$ & $-3.47978 \mathrm{E}-2$ & $-3.75841 \mathrm{E}-2$ & $5.56369 \mathrm{E}-2$ & $1.91593 \mathrm{E}-2$ & $-2.75063 \mathrm{E}-2$ \\
$i=5$ & $-4.92450 \mathrm{E}-2$ & $-4.46316 \mathrm{E}-2$ & $7.19123 \mathrm{E}-2$ & $2.38135 \mathrm{E}-2$ & $-3.55769 \mathrm{E}-2$ \\
$i=6$ & $-2.51385 \mathrm{E}-3$ & $3.37337 \mathrm{E}-3$ & $-1.61833 \mathrm{E}-3$ & $3.64315 \mathrm{E}-3$ & $-2.90671 \mathrm{E}-3$ \\
$i=7$ & $3.68866 \mathrm{E}-2$ & $2.53148 \mathrm{E}-2$ & $-5.16877 \mathrm{E}-2$ & $-8.55152 \mathrm{E}-3$ & $2.08971 \mathrm{E}-2$ \\
$i=8$ & $5.80492 \mathrm{E}-3$ & $-1.04420 \mathrm{E}-2$ & $-3.04257 \mathrm{E}-5$ & $3.95689 \mathrm{E}-3$ & $-2.15731 \mathrm{E}-4$ \\
$i=9$ & $-2.02777 \mathrm{E}-2$ & $-1.85917 \mathrm{E}-2$ & $3.19260 \mathrm{E}-2$ & $7.62300 \mathrm{E}-3$ & $-1.40661 \mathrm{E}-2$ \\
$i=10$ & $-8.44178 \mathrm{E}-3$ & $-2.77226 \mathrm{E}-3$ & $1.11309 \mathrm{E}-2$ & $1.36109 \mathrm{E}-3$ & $-5.00155 \mathrm{E}-3$ \\
\hline
\end{tabular}


Table 2: Coefficients $b_{i}$

\begin{tabular}{rr}
\hline & \multicolumn{1}{c}{$b_{i}$} \\
\hline$i=0$ & $4.50861 \mathrm{E}-2$ \\
$i=1$ & $1.13078 \mathrm{E}-3$ \\
$i=2$ & $3.12104 \mathrm{E}-3$ \\
$i=3$ & $8.64302 \mathrm{E}-4$ \\
$i=4$ & $1.57214 \mathrm{E}-2$ \\
$i=5$ & $8.16962 \mathrm{E}-2$ \\
$i=6$ & $7.84921 \mathrm{E}-2$ \\
$i=7$ & $-6.80863 \mathrm{E}-2$ \\
$i=8$ & $-9.79967 \mathrm{E}-2$ \\
$i=9$ & $2.04907 \mathrm{E}-2$ \\
$i=10$ & $3.66713 \mathrm{E}-2$ \\
\hline
\end{tabular}




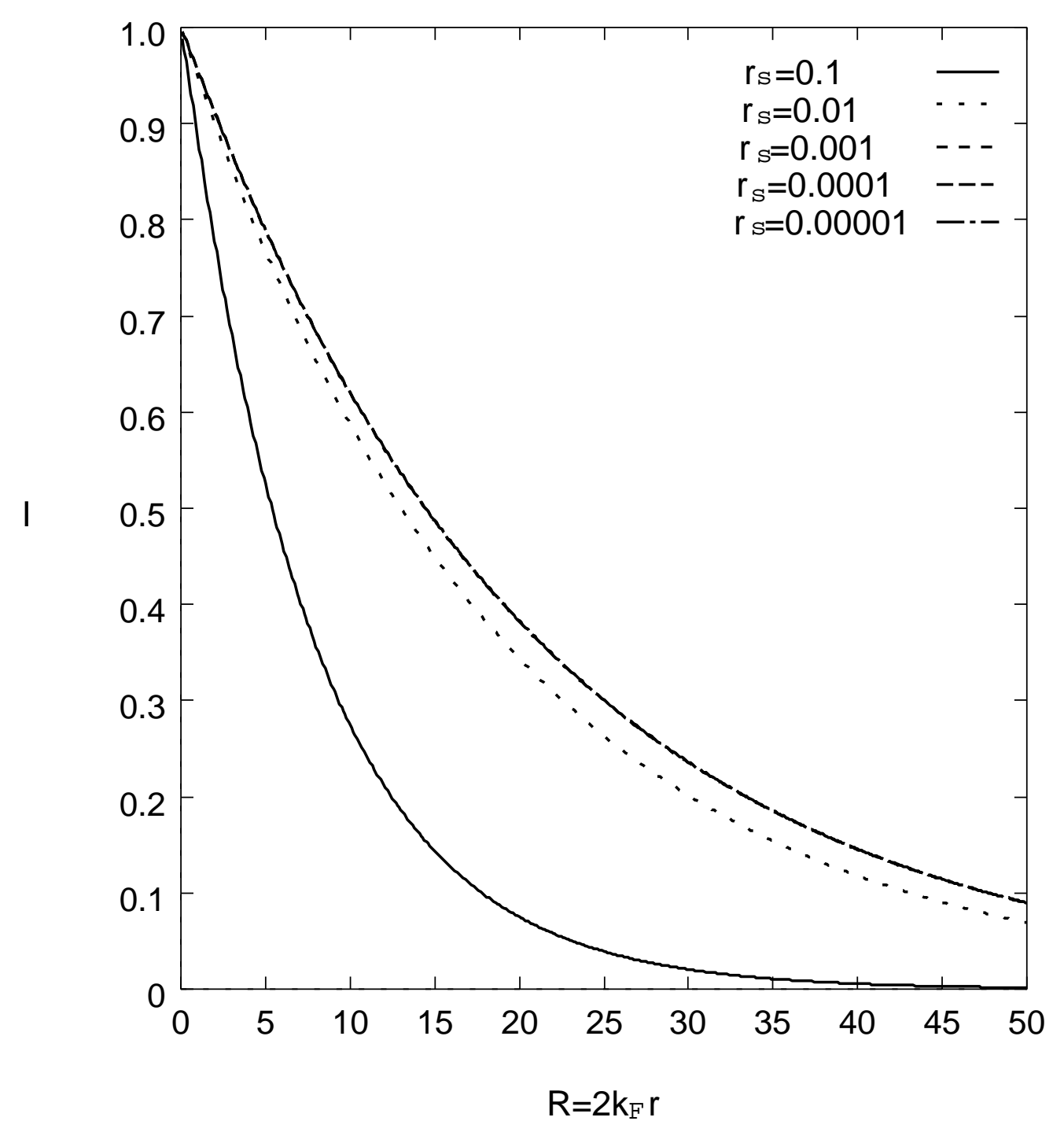

Fig. 1. $-I$ as a function of $R=2 k_{F} r$ for $r_{s}=0.1,0.01,0.001,0.0001,0.00001$. 


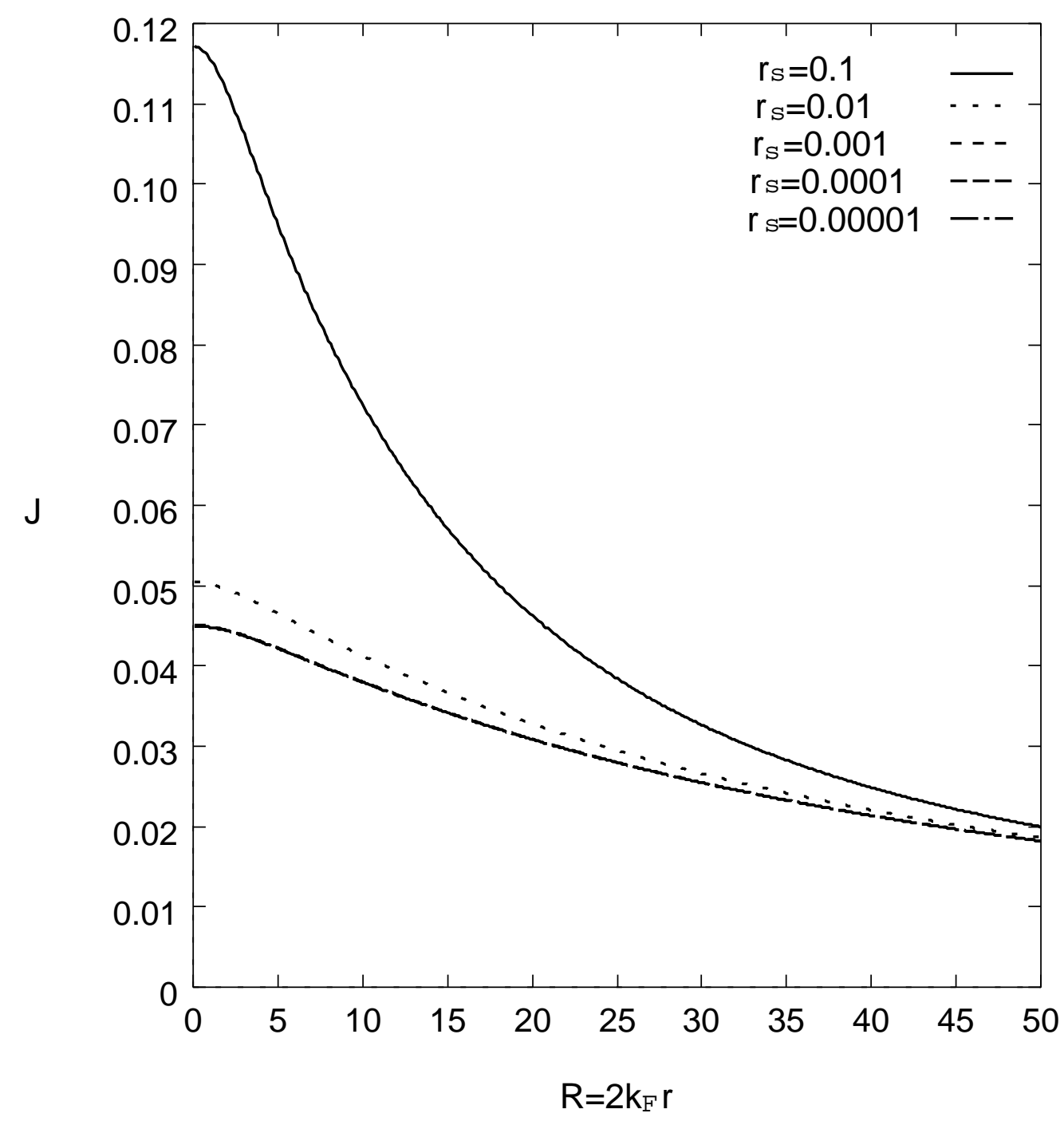

Fig. 2. $-J$ as a function of $R=2 k_{F} r$ for $r_{s}=0.1,0.01,0.001,0.0001,0.00001$. 


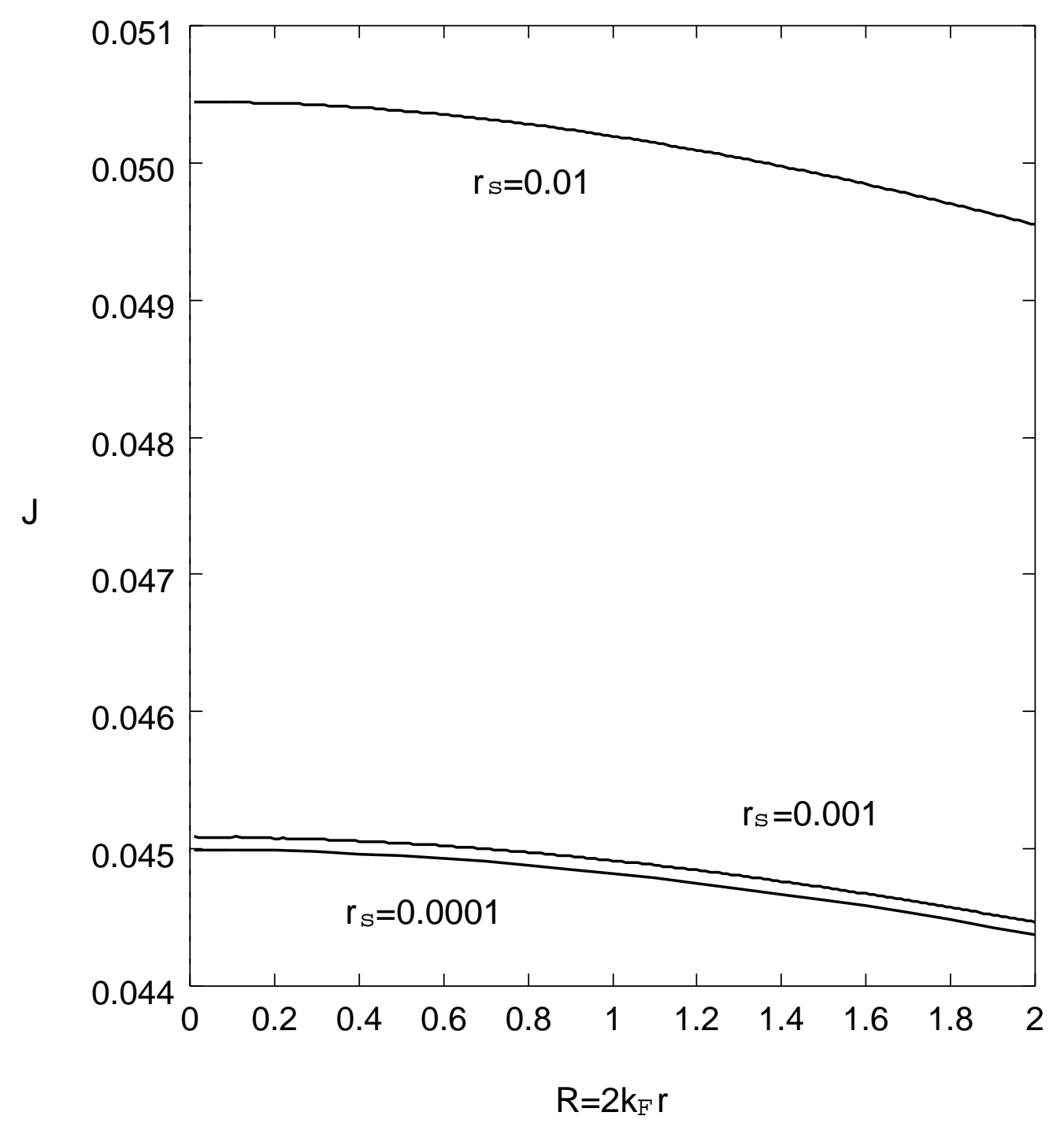

Fig. 3.- Same as Fig. 2, but for small values of $R=2 k_{F} r$. 


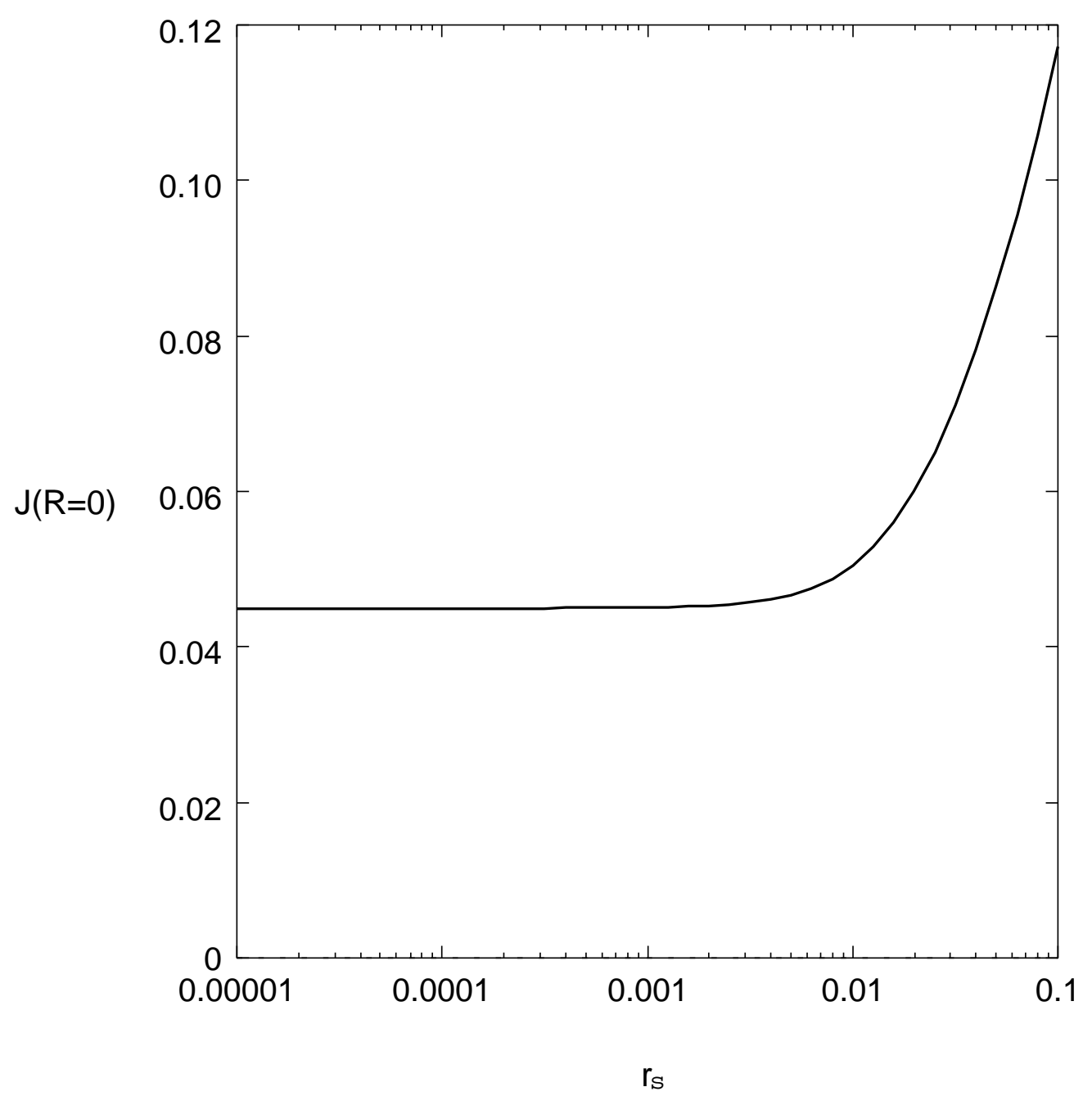

Fig. 4. $-J(R=0)$ as a function of $r_{s}$. 